\title{
Stable controllers for robust stabilization of systems with infinitely many unstable poles ${ }^{\text {th }}$
}

\author{
Masashi Wakaiki ${ }^{\mathrm{a}, *}$, Yutaka Yamamoto ${ }^{\mathrm{a}}$, Hitay Özbay ${ }^{\mathrm{b}}$ \\ a Department of Applied Analysis and Complex Dynamical Systems, Graduate School of Informatics, Kyoto University, Kyoto 606-8501, Japan \\ ${ }^{\mathrm{b}}$ Department of Electrical and Electronics Engineering, Bilkent University, Bilkent, Ankara TR-06800, Turkey
}

\section{A R T I C L E I N F O}

\section{Article history:}

Received 29 September 2012

Received in revised form

3 February 2013

Accepted 4 February 2013

Available online 18 April 2013

\section{Keywords:}

Strong stabilization

Robust stabilization

Infinite dimensional systems

$\mathscr{H}^{\infty}$ control

\begin{abstract}
A B S T R A C T
This paper studies the problem of robust stabilization by a stable controller for a linear time-invariant single-input single-output infinite dimensional system. We consider a class of plants having finitely many simple unstable zeros but possibly infinitely many unstable poles. First we show that the problem can be reduced to an interpolation-minimization by a unit element. Next, by the modified Nevanlinna-Pick interpolation, we obtain both lower and upper bounds on the multiplicative perturbation under which the plant can be stabilized by a stable controller. In addition, we find stable controllers to provide robust stability. We also present a numerical example to illustrate the results and apply the proposed method to a repetitive control system.
\end{abstract}

(C) 2013 Elsevier B.V. All rights reserved.

\section{Introduction}

In this paper, we study robust stabilization by a stable controller for a single-input single-output infinite dimensional system. The advantage of stable controllers is well appreciated in that such controllers are robust against a sensor or actuator failure [1] and the saturation of the control input [2]. Typical examples are flexible structures [3] and traffic networks [2]. Additionally, stable controllers are preferred for control of electromechanical positioning devices [4]. We also recall that two plants are simultaneously stabilizable if and only if an associated plant derived from these two plants is stabilizable by a stable controller [5].

For finite dimensional systems, several design methods of stable $\mathscr{H}^{\infty}$ controllers have been developed: linear matrix inequalities or algebraic Riccati equations [6,7] and non-smooth, non-convex optimization [8]. On the other hand, for infinite dimensional systems, while sensitivity reduction by a stable controller has been studied in [9-11], robust stabilization by a stable controller still remains to be an open problem.

Let us briefly summarize the difference between these two problems. Sensitivity reduction by a stable controller can be transformed to the modified Nevanlinna-Pick interpolation [9,12-14], and the associated $\mathscr{H}^{\infty}$-norm condition is $\|F\|_{\infty}<\rho$, where $F$ is

\footnotetext{
A shortened version of this paper was presented at the MTNS 2012.

* Corresponding author. Tel.: +81 75753 5904; fax: +81 757535517.

E-mail addresses: wakaiki@acs.i.kyoto-u.ac.jp (M. Wakaiki), yy@i.kyoto-u.ac.jp (Y. Yamamoto), hitay@bilkent.edu.tr (H. Özbay).
}

a solution of the unit interpolation problem. On the other hand, in robust stabilization by a stable controller, the counterpart is $\|W-m F\|_{\infty}<\rho$, where $W, 1 / W \in \mathscr{H}^{\infty}$ and $m \in \mathscr{H}^{\infty}$ is inner. Since $F$ needs to be a unit element, we cannot change this norm condition to a simpler one, although we can in the usual robust stabilization problem. We overcome this difficulty by extending the technique of [14]. We will discuss this technique in Section 3.

This paper studies a class of plants having finitely many simple unstable zeros but possibly infinitely many unstable poles. An example of such plants is a system with delayed feedback such as repetitive control systems $[15,16]$. The objective of the present paper is to obtain lower and upper bounds on the multiplicative perturbation under which the plant can be stabilized by a stable controller. We also develop a design method of stable controllers achieving robust stability by the method of $[9,10]$.

The paper is organized as follows: Section 2 gives the statement of the robust stabilization problem with stable controllers. In Section 3, we obtain a sufficient condition for the problem and find stable controllers for robust stabilization. A necessary condition follows along similar lines. We present a numerical example and apply the proposed method to a repetitive control system in Section 4.

\section{Notation and definitions}

Let $\mathbb{C}_{+}$denote the open right half-plane $\{s \in \mathbb{C} \mid \operatorname{Re} s>0\}$. For $s \in \mathbb{C} \backslash\{0\}$, the principal value $\log s$ is the complex logarithm whose imaginary part lies in the interval $(-\pi, \pi]$. 


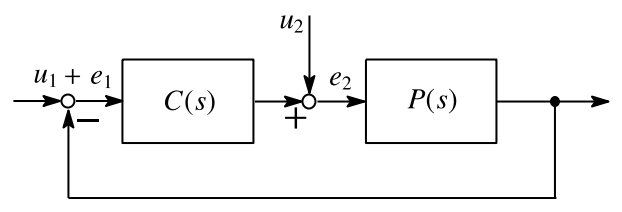

Fig. 1. Closed-loop system.

The space $\mathscr{H}^{\infty}$ denotes the Hardy space of functions that are bounded and analytic in $\mathbb{C}_{+}$, and $\mathcal{R} \mathscr{H}^{\infty}$ denotes the subset of $\mathscr{H}^{\infty}$ consisting of real-rational functions. $U \in \mathscr{H}^{\infty}$ is called a unit element in $\mathscr{H}^{\infty}$ if $U, 1 / U \in \mathcal{H}^{\infty}$. For $G \in \mathscr{H}^{\infty}$, the $\mathscr{H}^{\infty}$ norm is defined as $\|G\|_{\infty}:=\sup _{s \in \mathbb{C}_{+}}|G(s)|$. The field of fractions of $\mathscr{H}^{\infty}$ is denoted by $\mathcal{F}^{\infty}$.

Two functions $N, D \in \mathscr{H}^{\infty}$ are strongly coprime in the sense of [17] if $N X+D Y=1$ for some $X, Y \in \mathscr{H}^{\infty}$. By the corona theorem [5], $N$ and $D$ are strongly coprime if and only if there exists $\delta>0$ such that $|N(s)|+|D(s)| \geq \delta$ for all $s \in \mathbb{C}_{+}$.

To denote the interpolation data $G\left(s_{i}\right)=\alpha_{i}(i=1, \ldots, n)$ for $G \in \mathscr{H}^{\infty}$, we use the notation $\left(s_{i} ; \alpha_{i}\right)_{i=1}^{n}$.

\section{Problem statement}

Consider the linear, continuous-time, time-invariant, singleinput single-output closed-loop system given in Fig. 1. Let the plant $P$ and the controller $C$ belong to $\mathcal{F}^{\infty}$. $P$ is said to be stabilizable if there exists $C$ such that $S:=1 /(1+P C), C S$, and $P S$ belong to $\mathscr{H}^{\infty}$. For a given $P$, the set of all $C$ leading to $S, C S, P S \in \mathscr{H}^{\infty}$ is denoted by $\mathscr{C}(P)$. $P$ is strongly stabilizable if $\mathscr{H}^{\infty} \cap \mathscr{C}(P) \neq \emptyset$. We say that $C$ stabilizes $P$ if $C \in \mathscr{C}(P)$, and that $C$ strongly stabilizes $P$ if $C \in \mathscr{H}^{\infty} \cap \mathscr{C}(P)$.

Let $P$ be a real-rational proper function. Then $P$ is stabilizable by $C \in \mathcal{R} \mathscr{H}^{\infty}$ if and only if $P$ has the parity interlacing property [18]. On the other hand, if we do not require $C \in \mathcal{R} \mathscr{H}^{\infty}$ but $C \in \mathscr{H}^{\infty}$ allowing complex coefficients, every stabilizable $P \in \mathcal{F}^{\infty}$ is strongly stabilizable [19], via a complex-valued controller in general.

We make the following assumption on the plant throughout this paper:

Assumption 2.1. $P \in \mathcal{F}^{\infty}$ can be factorized into the following form:

$P=\frac{M_{n}}{M_{d}} N_{o}$,

where $M_{d} \in \mathscr{H}^{\infty}, M_{n} \in \mathcal{R} \mathscr{H}^{\infty}$ are inner functions and $N_{o}, 1 / N_{o} \in$ $\mathscr{H}^{\infty}$. We assume that $M_{n}$ possesses simple zeros $z_{1}, \ldots, z_{n}$ only and that $M_{d}, M_{n}$ are strongly coprime.

Under Assumption 2.1, $P$ has only finitely many unstable zeros arising from $M_{n}$, but $P$ is allowed to possess infinitely many unstable poles arising from $M_{d}$. In [20], it is shown how to factorize retarded or neutral time delay systems into the form (1) under some mild conditions.

Let $P$ be the nominal model of the plant. In this paper, we assume that the transfer function of the actual plant belongs to the following model set with multiplicative perturbations:

$\mathscr{P}_{\rho}:=\left\{P_{\Delta}=(1+W \Delta) P: \Delta \in \mathscr{H}^{\infty},\|\Delta\|_{\infty}<1 / \rho\right\}$

for some $\rho>0$.

Recall that the controller $C$ stabilizes all $P_{\Delta} \in \mathscr{P}_{\rho}$ if and only if $C$ stabilizes the nominal model $P$ and satisfies

$\|W T\|_{\infty} \leq \rho, \quad$ where $T:=\frac{P C}{1+P C}$.

See, e.g., $[1,5,21]$ for details.

We impose the following assumption on the weighting function:

Assumption 2.2. Both $W$ and $1 / W$ belong to $\mathscr{H}^{\infty}$.
Then robust stabilization by a stable controller can be formulated as follows:

Problem 2.3. Let Assumptions 2.1 and 2.2 hold. Suppose $\rho>0$. Determine whether there exists a controller $C \in \mathscr{H}^{\infty} \cap \mathscr{C}(P)$ satisfying (2). Also, if one exists, find such a controller $C$.

We call Problem 2.3 strong and robust stabilization. Our aim is to provide both a sufficient and a necessary condition for strong and robust stabilization. These conditions give lower and upper bounds on the multiplicative perturbation.

\section{Strong and robust stabilization}

In this section, we first transform Problem 2.3 to the problem of an interpolation-minimization by a unit element in $\mathscr{H}^{\infty}$. Next we obtain a sufficient condition as well as a necessary condition for the interpolation-minimization problem using the modified Nevanlinna-Pick interpolation [22].

Lemma 3.1 below is a scalar version of Lemma III.1 of [11]. This result provides a necessary and sufficient condition that a controller strongly stabilizes the plant. The next statement is different from that of Lemma III.1 in [11], but the modification is easy. So we omit the proof.

Lemma 3.1 ([11]). Suppose $P=N / D$, where $N, D \in \mathscr{H}^{\infty}$ are strongly coprime. Then $C$ strongly stabilizes $P$ if and only if $C, 1 /(D+$ NC) $\in \mathscr{H}^{\infty}$.

The following result shows that Problem 2.3 can be reduced to an interpolation-minimization by a unit element.

Theorem 3.2. Consider Problem 2.3 under Assumptions 2.1 and 2.2. Problem 2.3 is solvable if and only if there exists a function $F$ such that

$F, 1 / F \in \mathcal{H}^{\infty}$,

$\left\|W-M_{d} F\right\|_{\infty} \leq \rho$,

$F\left(z_{i}\right)=\frac{W\left(z_{i}\right)}{M_{d}\left(z_{i}\right)}, \quad i=1, \ldots, n$.

Furthermore, once such a function $F$ is constructed, the solution of Problem 2.3 is given by

$C=\frac{W-M_{d} F}{M_{n} N_{o} F}$.

Proof (Necessity). Let $C$ be a solution of Problem 2.3. Define $F:=$ $W /\left(M_{d}+M_{n} N_{o} C\right)$. Then $F$ satisfies (3) by Lemma 3.1. Since

$W T=W\left(1-\frac{M_{d} F}{W}\right)=W-M_{d} F$,

$F$ also achieves the norm constraint (4). In addition,

$F\left(z_{i}\right)=\frac{W\left(z_{i}\right)}{M_{d}\left(z_{i}\right)+M_{n}\left(z_{i}\right) N_{o}\left(z_{i}\right) C\left(z_{i}\right)}=\frac{W\left(z_{i}\right)}{M_{d}\left(z_{i}\right)}, \quad i=1, \ldots, n$.

Thus $F$ satisfies (3)-(5).

(Sufficiency). Suppose $F$ satisfies (3)-(5), and define $C$ by (6).

We show $C \in \mathscr{H}^{\infty}$ as follows. Since $1 / N_{o}, 1 / F \in \mathscr{H}^{\infty}$, it follows from (6) that

$M_{n} C=\frac{W-M_{d} F}{N_{o} F} \in \mathscr{H}^{\infty}$.

Suppose $C \notin \mathscr{H}^{\infty}$. Then the unstable poles of $C$ must be the zeros of $M_{n}$ by (8). Let $z_{i}$ be such a pole. Since the zeros of $M_{n}$ are simple, 
it follows that $\left(M_{n} C\right)\left(z_{i}\right) \neq 0$. In addition, since the units $N_{o}$ and $F$ do not have unstable zeros, $N_{o}\left(z_{i}\right) \neq 0$ and $F\left(z_{i}\right) \neq 0$. Hence

$W\left(z_{i}\right)-M_{d}\left(z_{i}\right) F\left(z_{i}\right)=\left(M_{n} C\right)\left(z_{i}\right) \cdot N_{o}\left(z_{i}\right) F\left(z_{i}\right) \neq 0$,

which contradicts (5). Thus $C$ belongs to $\mathscr{H}^{\infty}$.

Moreover since

$\frac{1}{M_{d}+M_{n} N_{o} C}=\frac{W}{F} \in \mathscr{H}^{\infty}$,

$C$ strongly stabilizes $P$ by Lemma 3.1. $C$ also achieves the norm constraint (2) by (4) and (7). Thus $C$ is a solution of Problem 2.3.

We obtain a sufficient condition as well as a necessary condition for robust stabilizability by a stable controller using the following problem:

Problem 3.3 ([22,23]). Suppose $s_{1}, \ldots, s_{n} \in \mathbb{C}_{+}$are distinct, and let $\beta_{1}, \ldots, \beta_{n} \in \mathbb{C} \backslash\{0\}$. Determine whether there exists a function $G$ such that $G, 1 / G \in \mathscr{H}^{\infty},\|G\|_{\infty} \leq 1$, and $G\left(s_{i}\right)=\beta_{i}$ for $i=1, \ldots, n$. Also, if one exists, find such a function $G$.

Problem 3.3 is called the modified Nevanlinna-Pick interpolation problem [22].

The difference between Problem 3.3 and the Nevanlinna-Pick interpolation problem $[1,21]$ is that Problem 3.3 has the condition $1 / G \in \mathscr{H}^{\infty}$. Despite this difference, the solvability of Problem 3.3 is also equivalent to the positive semi-definiteness of an associated Pick matrix.

Theorem 3.4 ([22,23]). Consider Problem 3.3. Define $\alpha_{i}:=\phi\left(s_{i}\right)$ for all $i=1, \ldots, n$, where the conformal map $\phi$ is

$\phi: \mathbb{C}_{+} \rightarrow \mathbb{D}: s \mapsto \frac{s-1}{s+1}$.

Problem 3.3 is solvable if and only if there exists an integer set $\left\{k_{1}, \ldots, k_{n}\right\}$ such that the Pick matrix $\mathbf{P}\left(\left\{k_{1}, \ldots, k_{n}\right\}\right)$,

$\mathbf{P}\left(\left\{k_{1}, \ldots, k_{n}\right\}\right):=\left[\frac{-\log \beta_{p}-\log \overline{\beta_{q}}+j 2 \pi\left(k_{q}-k_{p}\right)}{1-\alpha_{p} \overline{\alpha_{q}}}\right]_{p, q=1}^{n}$

is positive semi-definite.

The next result gives a solution of Problem 3.3 by the Nevanlinna-Pick interpolation.

Theorem 3.5 ([9,10]). Consider Problem 3.3. Fix $\sigma>0$. Define $\alpha_{i}$ in the same way as in Theorem 3.4 and $\zeta_{i}:=\Psi_{\sigma}\left(-\log \beta_{i}-j 2 \pi k_{i}\right)$ for $i=1, \ldots, n$, where $\left\{k_{1}, \ldots, k_{n}\right\}$ is an integer set and the conformal $\operatorname{map} \Psi_{\sigma}$ is

$\Psi_{\sigma}:\left\{s \in \mathbb{C}_{+}: 0<\operatorname{Re} s<\sigma\right\} \rightarrow \mathbb{D}: s \mapsto \frac{j e^{-j \pi s / \sigma}-1}{j e^{-j \pi s / \sigma}+1}$.

If there exists an analytic function $g: \mathbb{D} \rightarrow \mathbb{D}$ such that $g\left(\alpha_{i}\right)=\zeta_{i}$ for $i=1, \ldots, n$, then

$G(s):=\exp \left(-\frac{\sigma}{2}-\frac{j \sigma}{\pi} \log \left(\frac{1+g(\phi(s))}{1-g(\phi(s))}\right)\right)$

is a solution to Problem 3.3.

Remark 3.6. 1. In Theorem 3.4, we have an infinite number of $\mathbf{P}\left(\left\{k_{1}, \ldots, k_{n}\right\}\right)$. Note, however, that in order that $\mathbf{P}\left(\left\{k_{1}, \ldots\right.\right.$, $\left.\left.k_{n}\right\}\right)$ be positive semi-definite it is necessary that $K_{p q}:=k_{p}-$ $k_{q}$ be bounded. It turns out that only finitely many distinct $\mathbf{P}\left(\left\{k_{1}, \ldots, k_{n}\right\}\right)$ could possibly be positive semi-definite. In fact, for the positive semi-definiteness of $\mathbf{P}\left(\left\{k_{1}, \ldots, k_{n}\right\}\right), K_{p q}$ must satisfy the following quadratic inequality:

$$
\begin{aligned}
& \operatorname{det}\left[\begin{array}{cc}
\frac{-\log \beta_{p}-\log \overline{\beta_{p}}}{1-\alpha_{p} \overline{\alpha_{p}}} & \frac{-\log \beta_{p}-\log \overline{\beta_{q}}-j 2 \pi K_{p q}}{1-\alpha_{p} \overline{\alpha_{q}}} \\
\frac{-\log \beta_{q}-\log \overline{\beta_{p}}+j 2 \pi K_{p q}}{1-\alpha_{q} \overline{\alpha_{p}}} & \frac{-\log \beta_{q}-\log \overline{\beta_{q}}}{1-\alpha_{q} \overline{\alpha_{q}}}
\end{array}\right] \\
& =a K_{p q}^{2}+b K_{p q}+c \geq 0,
\end{aligned}
$$

where $a:=-4 \pi^{2}, b:=4 \pi \operatorname{Re}\left[j\left(-\log \beta_{p}-\log \overline{\beta_{q}}\right)\right]$, and

$$
\begin{aligned}
c:= & \left(\frac{\log \beta_{p}+\log \overline{\beta_{p}}}{1-\alpha_{p} \overline{\alpha_{p}}} \cdot \frac{\log \beta_{q}+\log \overline{\beta_{q}}}{1-\alpha_{q} \overline{\alpha_{q}}}\right. \\
& \left.-\left|\frac{\log \beta_{p}+\log \overline{\beta_{q}}}{1-\alpha_{p} \overline{\alpha_{q}}}\right|^{2}\right) \cdot\left|1-\alpha_{p} \overline{\alpha_{q}}\right|^{2} .
\end{aligned}
$$

Hence $D:=b^{2}-4 a c \geq 0$ and $(b+\sqrt{D}) /(2 a) \leq K_{p q} \leq(b-\sqrt{D}) /$ (2a). Thus we can check the solvability of Problem 3.3 in a finite number of steps. See $[23,24]$ for the details.

2. A function $f$ is said to be real if $\overline{f(s)}=f(\bar{s})$. Simple calculations show that $G(s)$ in (10) is real if $g(z)=j \cdot g_{0}(z)$, where $g_{0}(z)$ is real.

For finite dimensional systems [12-14] and systems with infinitely many unstable modes $[9,10]$, the problem of sensitivity reduction by a stable controller is equivalent to Problem 3.3. On the other hand, the difficulty of strong and robust stabilization is the $\mathscr{H}^{\infty}$-norm condition (4) in Theorem 3.2.

We now develop both a sufficient and a necessary condition for (4). It follows from these conditions that we obtain lower and upper bounds on the perturbation by Problem 3.3. Theorem 3.4 and Remark 3.6.1 show that we can compute these bounds by calculations of the finitely many Pick matrices. Additionally, we find stable controllers for robust stabilization by Theorem 3.5.

Define $\rho_{\text {inf }}:=\inf _{C \in H^{\infty} \cap \mathscr{C}(P)}\|W T\|_{\infty}$. Then $K_{\text {sup }}:=1 / \rho_{\text {inf }}$ can be regarded as the largest allowable multiplicative uncertainty bound for robust stability with a stable controller. Theorem 3.7 below gives a lower bound of $K_{\text {sup }}$ and stable robust controllers.

Theorem 3.7. Consider Problem 2.3 under Assumptions 2.1 and 2.2. Suppose $\|W\|_{\infty}<\rho$. Choose $W_{s}$ satisfying $W_{s}, 1 / W_{s} \in \mathcal{R} \mathcal{H}^{\infty}$ and $\left|W_{s}(j \omega)\right| \leq \rho-|W(j \omega)|$ for almost all $\omega \in \mathbb{R}$. Define $\beta_{i}:=W\left(z_{i}\right) /$ $\left(M_{d}\left(z_{i}\right) W_{s}\left(z_{i}\right)\right)$ for $i=1, \ldots, n$. If $G$ is a solution of Problem 3.3 with the interpolation data $\left(z_{i} ; \beta_{i}\right)_{i=1}^{n}$, then $K_{\text {sup }} \geq 1 / \rho$ and

$C:=\frac{W-M_{d} W_{s} G}{M_{n} N_{0} W_{s} G}$

is a solution to Problem 2.3.

Proof. Note that $\beta_{i} \neq 0$ for each $i$ because the unit $W$ does not have unstable zeros. By Theorem 3.2, it suffices to show that there exists $F$ satisfying (3)-(5).

Let us first obtain a sufficient condition for (4). Since $M_{d}$ is inner,

$\left|W(j \omega)-M_{d}(j \omega) F(j \omega)\right| \leq\left|M_{d}(j \omega)\right| \cdot|F(j \omega)|+|W(j \omega)|$

$$
\leq|F(j \omega)|+\rho-\left|W_{\mathrm{s}}(j \omega)\right|
$$

for almost all $\omega \in \mathbb{R}$. Moreover $|F(j \omega)|+\rho-\left|W_{s}(j \omega)\right| \leq \rho$ if and only if $\left|\left(F / W_{s}\right)(j \omega)\right| \leq 1$. It follows that if $\left\|F / W_{s}\right\|_{\infty} \leq 1$, then we have (4).

Suppose $G$ is a solution of Problem 3.3 with $\left(z_{i} ; \beta_{i}\right)_{i=1}^{n}$. Define $F:=W_{s} G$. By the argument given above, $F$ achieves $(4)$ because $\left\|F / W_{\mathrm{s}}\right\|_{\infty}=\|G\|_{\infty} \leq 1$. Since $G$ and $W_{\mathrm{s}}$ are unit elements, $F$ satisfies (3). Moreover the interpolation conditions (5) can be obtained directly by those of $G$. Thus $F$ satisfies (3)-(5). By substituting $F=W_{\mathrm{s}} G$ into (6), we can also derive (11). 
In the same way, an upper bound of $K_{\text {sup }}$ can be obtained by the next result:

Theorem 3.8. Consider Problem 2.3 under Assumptions 2.1 and 2.2. Choose $W_{n}$ satisfying $W_{n}, 1 / W_{n} \in \mathcal{R} \mathscr{H}^{\infty}$ and $\left|W_{n}(j \omega)\right| \geq \rho+$ $|W(j \omega)|$ for almost all $\omega \in \mathbb{R}$. Define $\gamma_{i}:=W\left(z_{i}\right) /\left(M_{d}\left(z_{i}\right) W_{n}\left(z_{i}\right)\right)$ for $i=1, \ldots, n$. If Problem 3.3 with the interpolation data $\left(z_{i} ; \gamma_{i}\right)_{i=1}^{n}$ is not solvable, then $K_{\text {sup }} \leq 1 / \rho$.

Proof. As in the proof of Theorem 3.7, we can derive a necessary condition for (4) by $\left|W(j \omega)-M_{d}(j \omega) F(j \omega)\right| \geq|F(j \omega)|+\rho-$ $\left|W_{\mathrm{n}}(j \omega)\right|$ for almost all $\omega \in \mathbb{R}$. The rest of the proof follows the same lines as that of Theorem 3.7, so it is omitted.

Remark 3.9. 1. In Assumption 2.1, we have taken a biproper plant having infinitely many unstable poles as the nominal model. Therefore the condition $\|W\|_{\infty}<\rho$ in Theorem 3.7 implies that the controllers obtained by our proposed method may not robustly stabilize strictly proper plants. In the first place, however, we should pose the question: Are strictly proper plants with infinitely many unstable poles stabilizable? The answer is negative; see Appendix.

2. By the MATLAB command fitmagfrd, we can compute $W_{\mathrm{s}}, W_{\mathrm{n}}$ in Theorems 3.7 and 3.8.

Theorem 3.7 generally gives an infinite dimensional controller. A natural question at this stage is the following: Does a finite dimensional controller that approximates the derived controller stabilize the plant and satisfy the $\mathscr{H}^{\infty}$-norm condition (2)? Rational approximations can be obtained from the frequency response data with approximation methods for stable infinite dimensional systems; see, e.g., [25] and its references.

To ensure that the approximation $C_{a} \in \mathcal{R} \mathscr{H}^{\infty}$ still stabilizes the plant, we can obtain an error bound on the difference $\| C-$ $C_{a} \|_{\infty}[12$, Lemma 4].

Define

$T_{a}:=\frac{P C_{a}}{1+P C_{a}}$.

The following result illustrates that we can also obtain an upper bound of $\left\|W T_{a}\right\|_{\infty}$ by $\left\|C-C_{a}\right\|_{\infty}$.

Proposition 3.10. Let $P \in \mathcal{F}^{\infty}$ and $W \in \mathscr{H}^{\infty}$. Suppose there exists $C \in \mathscr{H}^{\infty} \cap \mathscr{C}(P)$ and $C_{a} \in \mathcal{R} \mathscr{H}^{\infty} \cap \mathscr{C}(P)$. Define $\delta:=\|P /(1+P C)\|_{\infty}$ and $\epsilon:=\left\|C-C_{a}\right\|_{\infty}$. If $\delta \epsilon<1$, then

$\left\|W T_{a}\right\|_{\infty} \leq \frac{\delta \epsilon \cdot\|W\|_{\infty}+\|W T\|_{\infty}}{1-\delta \epsilon}$,

where $T$ and $T_{a}$ are defined by (2) and (12) respectively.

Proof. Routine calculations show that

$T-T_{a}=\frac{P}{1+P C}\left(1-T_{a}\right)\left(C-C_{a}\right)$.

Hence

$$
\begin{aligned}
\left\|W T-W T_{a}\right\|_{\infty} & \leq \delta \epsilon \cdot\left\|W\left(1-T_{a}\right)\right\|_{\infty} \\
& \leq \delta \epsilon \cdot\left(\|W\|_{\infty}+\left\|W T_{a}\right\|_{\infty}\right) .
\end{aligned}
$$

Since $\left\|W T_{a}\right\|_{\infty}-\|W T\|_{\infty} \leq\left\|W T-W T_{a}\right\|_{\infty}$, it follows from (14) that

$(1-\delta \epsilon) \cdot\left\|W T_{a}\right\|_{\infty} \leq \delta \epsilon \cdot\|W\|_{\infty}+\|W T\|_{\infty}$.

Thus we obtain (13) if $\delta \epsilon<1$.

\section{Numerical examples}

In this section, we present a numerical example to show the effectiveness of the results. We also apply the proposed method to

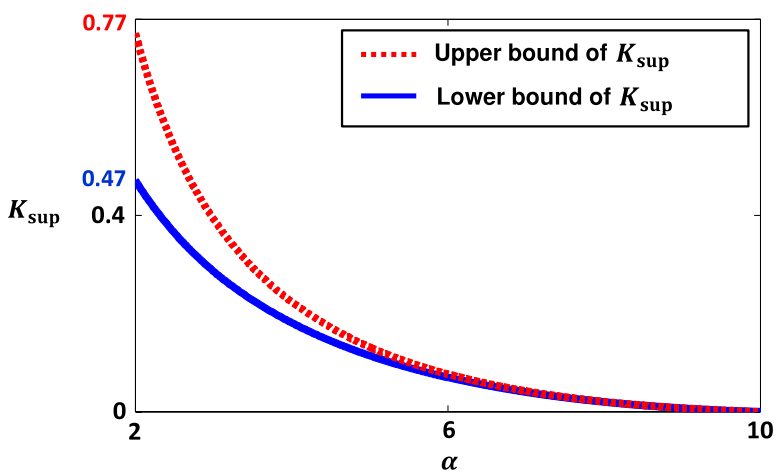

Fig. 2. The unstable zero $\alpha$ versus the supremum gain $K_{\text {sup }}$.

a repetitive control system $[15,16]$. Repetitive control attempts to track or reject arbitrary periodic signals of a fixed period. Tracking or disturbance rejection of periodic signals appears in many applications, e.g., disk drives [26] and industrial manipulators [27]

Example 1. Consider Problem 2.3 with the following infinite dimensional system $P$, weighting function $W$, and positive constant $\rho$ :

$P(s)=\frac{(s-\alpha)\left(s-4 e^{-s}+1\right)}{(s-10)(s-15)\left(2 e^{-s}+1\right)}$,

$W(s)=K \cdot \frac{s+1}{s+10}, \quad \rho=1$,

where $2 \leq \alpha<10$ and $K>0$. Let $p$ be the only root of $s-$ $4 e^{-s}+1=0$ in $\mathbb{C}_{+}$(note that $p \approx 0.7990$ ). Using the factorization method of [20], $P$ can be factorized as $P=M_{n} N_{o} / M_{d}$, where

$$
\begin{aligned}
M_{n}(s) & :=\frac{(s-\alpha)(s-p)}{(s+\alpha)(s+p)} \\
M_{d}(s) & :=\frac{(s-10)(s-15)\left(2 e^{-s}+1\right)}{(s+10)(s+15)\left(e^{-s}+2\right)} \\
N_{o}(s) & :=\frac{(s+\alpha)(s+p)\left(s-4 e^{-s}+1\right)}{(s-p)(s+10)(s+15)\left(e^{-s}+2\right)} .
\end{aligned}
$$

Let $K_{\text {sup }}$ be the supremum of $K$ such that there exists $C \in \mathcal{H}^{\infty} \cap$ $\mathscr{C}(P)$ satisfying (2). Fig. 2 shows the relationship between $\alpha$ and

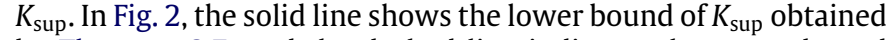
by Theorem 3.7, and the dashed line indicates the upper bound of $K_{\text {sup }}$ derived by Theorem 3.8. We compute both $W_{\mathrm{s}}$ and $W_{\mathrm{n}}$ in Theorems 3.7 and 3.8 by the MATLAB function fitmagfrd. Both lines in Fig. 2 decrease to 0 as $\alpha$ becomes closer to 10 . The reason for this drop is that an unstable pole-zero cancellation occurs in $P$ when $\alpha=10$.

Let $\alpha=2$. Then we obtain the lower bound 0.471 and the upper bound 0.771 . We also find a stable controller to achieve robust stability for $K=0.468$ by Theorem 3.5 with $\sigma=100$. See Fig. 3 of [9] for a discussion on the selection of $\sigma$ based on a specific numerical example.

When $K=0.468, W_{\mathrm{s}}$ in Theorem 3.7 and $g$ in Theorem 3.5 are given by

$W_{s}(s) \approx \frac{0.53(s+10.20)}{(s+5.86)}$,

$g(z)=j \cdot g_{0}(z), \quad$ where $g_{0}(z) \approx \frac{1.049 z+1}{z+1.050}$

The above $W_{s}$ is obtained by $\mathrm{f}$ itmagf $r \mathrm{~d}$. The stable controller that provides robust stability is obtained by (11), where $G(s)$ is defined in (10) with $g(z)$.

Note that $G(s)$ in (10) is real by Remark 3.6.2. A further investigation of $G$ is conducted through an example in [9]. 


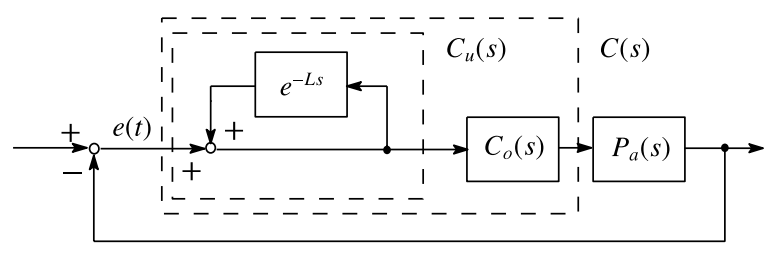

Fig. 3. Repetitive control system.

Example 2 (Application to Repetitive Control Systems). Consider the repetitive control system given in Fig. 3, where $L=1$ and $P_{a}$ belongs to the following model set:

$\mathscr{P}=\left\{P_{a}(s)=\frac{(s-6)(s-9)}{(a s+8)(s-5)}: 0.8 \leq a \leq 1.2\right\}$.

Note that the plant must be biproper for the exponential stability of the closed-loop system [16, Theorem 5.12]. When the plant is strictly proper, we need a modified repetitive controller $[15,16]$. See [28] for the details of robust stabilization of modified repetitive control systems.

The repetitive controller $C$ consists of two parts: $C_{u}$ and $C_{o}$. $C_{u}=1 /\left(1-e^{-L s}\right)$ is the internal model of any periodic signals with period $L$. The existence of such an internal model is equivalent to the exponential decay of the error $e(t)$ under the hypothesis of the exponential stability of the closed-loop system [16]. On the other hand, $C_{o}$ is designed for the desired performance. Our goal in this example is to determine whether there exists $C_{0} \in \mathcal{H}^{\infty}$ such that $C=C_{u} C_{o}$ stabilizes all $P_{a} \in \mathscr{P}$ and the error $e(t)$ tends exponentially to zero for any $P_{a} \in \mathscr{P}$.

For $\varepsilon>0$, let $\mathbb{C}_{-\varepsilon}$ denote $\{s \in \mathbb{C} \mid \operatorname{Re} s>-\varepsilon\}$ and let $\mathscr{H}^{\infty}\left(\mathbb{C}_{-\varepsilon}\right)$ denote the set of functions that are bounded and analytic in $\mathbb{C}_{-\varepsilon}$. For exponential stability, it is necessary and sufficient that $S, C S$, and $P S$ belong to $\mathscr{H}^{\infty}\left(\mathbb{C}_{-\varepsilon}\right)$ for some $\varepsilon>0$ [29, Theorem 3.1]. In addition, if $\varepsilon$ is sufficiently small, then

$\mathscr{P} \subset\left\{P_{\Delta}=(1+W \Delta) P_{1}: \Delta \in \mathscr{H}^{\infty}\left(\mathbb{C}_{-\varepsilon}\right), \sup _{s \in \mathbb{C}_{-\varepsilon}}|\Delta(s)|<1\right\}$,

where

$P_{1}(s):=\frac{(s-6)(s-9)}{(s-5)(s+8)}, \quad W(s)=\frac{0.25038(s+0.02384)}{s+10}$.

Now let us consider the closed-loop system in Fig. 4. By the preceding discussion, to determine whether there exists $C_{o} \in \mathscr{H}^{\infty}$ yielding the exponential stability of the closed-loop system for every $P_{a} \in \mathscr{P}$, we study Problem 2.3 with

$\tilde{P}(s):=P(s-\varepsilon)=C_{u}(s-\varepsilon) P_{1}(s-\varepsilon)$,

$\tilde{W}(s):=W(s-\varepsilon), \quad \rho:=1$.

Once we find a solution $\tilde{C}$ of this problem, $C_{o}(s):=\tilde{C}(s+\varepsilon) \in$ $\mathscr{H}^{\infty}\left(\mathbb{C}_{-\varepsilon}\right)$ makes the closed-loop system exponential stable for every $\Delta \in \mathscr{H}^{\infty}\left(\mathbb{C}_{-\varepsilon}\right)$ satisfying $\sup _{s \in \mathbb{C}_{-\varepsilon}}|\Delta(s)|<1$ in Fig. 4.

Let $\varepsilon=0.001$, which satisfies (15). $\tilde{P}$ in (16) can be factorized as $\tilde{P}=M_{n} N_{o} / M_{d}$, where

$M_{n}(s):=\frac{(s-\varepsilon-6)(s-\varepsilon-9)}{(s+\varepsilon+6)(s+\varepsilon+9)}$,

$M_{d}(s):=\frac{\left(1-e^{\varepsilon} e^{-s}\right)(s-\varepsilon-5)}{\left(e^{-s}-e^{\varepsilon}\right)(s+\varepsilon+5)}$,

$N_{o}(s):=\frac{(s+\varepsilon+6)(s+\varepsilon+9)}{\left(e^{-s}-e^{\varepsilon}\right)(s+\varepsilon+5)(s-\varepsilon+8)}$.

Define $\tilde{T}:=\tilde{P} \tilde{C} /(1+\tilde{P} \tilde{C})$. It follows from Theorems 3.7 and 3.8 that $0.71<\inf _{\tilde{C} \in \mathscr{H} \infty \cap \mathscr{C}(\tilde{P})}\|\tilde{W} \tilde{T}\|_{\infty}<0.97$. The MATLAB function fitmagfrd is used for $W_{\mathrm{s}}$ and $W_{\mathrm{n}}$ in Theorems 3.7 and 3.8.

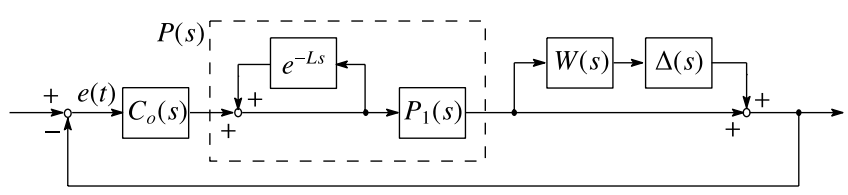

Fig. 4. Robust stabilization for the repetitive control system.

Thus there exists $C_{o} \in \mathscr{H}^{\infty}$ such that the repetitive controller $C=C_{u} C_{o}$ stabilizes all $P_{a} \in \mathscr{P}$ and achieves the exponential decay of $e(t)$ for any $P_{a} \in \mathscr{P}$.

\section{Concluding remarks}

We have studied the strong and robust stabilization problem for single-input single-output infinite dimensional systems. The plants we consider can have only finitely many simple unstable zeros but may possess infinitely many unstable poles. It still remains an open problem to obtain a necessary and sufficient condition for this robust stabilization problem. However, using the modified Nevanlinna-Pick interpolation, we have obtained both lower and upper bounds on the multiplicative perturbation under which a stable controller can stabilize the plant. Moreover we have found stable controllers to achieve robust stability. We have also presented a numerical example to illustrate the results. A repetitive control system has been discussed as an application of the proposed method.

\section{Appendix. Stabilizability of strictly proper plants having in- finitely many unstable poles}

We answer the question: Can a linear time-invariant controller stabilize a strictly proper plant with an infinite number of unstable poles?

The previous works $[30,31]$ on $\mathscr{H}^{\infty}$ control of plants with infinitely many unstable modes assume that the plants are biproper. In addition, a strictly proper neutral delay system is not stabilizable by a finite dimensional controller [32]. However the above question is not fully answered. Based on the Bezout identity, the next result shows that more general strictly proper plants with infinitely many unstable poles are not stabilizable in the sense of [17].

Proposition A.1. Let nonzero $N, D \in \mathcal{H}^{\infty}$ be weakly coprime in the sense of [17], i.e., every greatest common divisor of $N$ and $D$ is a unit element. Suppose D has infinitely many zeros in $\mathbb{C}_{+}$, and that the set of these unstable zeros has no limit points on the imaginary axis. If $N$ satisfies

$\lim _{R \rightarrow \infty} \sup _{|s|>R}|N(s)|=0$,

then $P:=N / D$ is not stabilizable.

Proof. Suppose $P$ is stabilizable. Then by Theorem 1 of [17], there exist $X, Y \in \mathscr{H}^{\infty}$ such that

$N(s) X(s)+D(s) Y(s)=1$ for all $s \in \mathbb{C}_{+}$.

By (A.1), for every $\varepsilon>0$, there exists $R>0$ such that $|N(s)|$. $\|X\|_{\infty}<\varepsilon$ for all $s \in \mathbb{C}_{+}$satisfying $|s|>R$. In addition, there exists $z_{0} \in \mathbb{C}_{+}$such that $D\left(z_{0}\right)=0$ and $\left|z_{0}\right|>R$. Otherwise the set of the unstable zeros of $D$ has at least one limit point in $\left\{s \in \mathbb{C}_{+}:|s| \leq R\right\}$, which implies that $D(s)=0$ for all $s \in \mathbb{C}_{+}$. Let $\varepsilon<1$. Then

$\left|N\left(z_{0}\right) X\left(z_{0}\right)+D\left(z_{0}\right) Y\left(z_{0}\right)\right| \leq\left|N\left(z_{0}\right)\right| \cdot\|X\|_{\infty}<\varepsilon<1$.

This contradicts (A.2). Thus $P$ is not stabilizable. 


\section{References}

[1] J.C. Doyle, B.A. Francis, A. Tannenbaum, Feedback Control Theory, Macmillan, New York, 1992

[2] H.U. Ünal, A. Iftar, Stable $\mathscr{H}^{\infty}$ flow controller design using approximation of FIR filters, Trans. Inst. Meas. Control 34 (2012) 3-25.

[3] A. Cavallo, G. De Maria, C. Natale, S. Pirozzi, Robust control of flexible structures with stable bandpass controllers, Automatica 44 (2008) 1251-1260.

[4] M. van de Wal, G. van Baars, F. Sperling, O. Bosgra, Multivariable $\mathscr{H}_{\infty} / \mu$ feedback control design for high-precision wafer stage motion, Control Eng. Pract. 10 (2002) 739-755.

[5] M. Vidyasagar, Control System Synthesis: A Factorization Approach, MIT Press, Cambridge, MA, 1985.

[6] S. Gümüssoy, H. Özbay, Remarks on strong stabilization and stable $\mathscr{H}^{\infty}$ controller design, IEEE Trans. Automat. Control 50 (2005) 2083-2087.

[7] I.R. Petersen, Robust $H^{\infty}$ control of an uncertain system via a stable output feedback controller, IEEE Trans. Automat. Control 54 (2009) 1418-1423.

[8] S. Gümüşsoy, M. Millstone, M.L. Overton, $\mathscr{H}^{\infty}$ strong stabilization via HIFOO, a package for fixed-order controller design, in: Proc. 47th IEEE CDC, 2008.

[9] S. Gümüşsoy, H. Özbay, Sensitivity minimization by strongly stabilizing controllers for a class of unstable time-delay systems, IEEE Trans. Automat. Control 54 (2009) 590-595.

[10] H. Özbay, Stable $\mathscr{H}^{\infty}$ controller design for systems with time delays, in: Perspectives in Mathematical System Theory, Control, and Signal Processing, Springer-Verlag, 2010, pp. 105-113.

[11] M. Wakaiki, Y. Yamamoto, H. Özbay, Sensitivity reduction by strongly stabilizing controllers for MIMO distributed parameter systems, IEEE Trans. Automat. Control 57 (2012) 2089-2094.

[12] C. Ganesh, J.B. Pearson, Design of optimal control systems with stable feedback, in: Proc. ACC 1986, 1986.

[13] H. Ito, H. Ohmori, A. Sano, Design of stable controllers attaining low $\mathscr{H}^{\infty}$ weighted sensitivity, IEEE Trans. Automat. Control 38 (1993) 485-488.

[14] S. Hara, M. Vidyasagar, Sensitivity minimization and robust stabilization by stable controller SISO systems, in: Proc. MTNS 1989, 1989.

[15] S. Hara, Y. Yamamoto, T. Omata, M. Nakano, Repetitive control system: a new type servo system for periodic exogenous signals, IEEE Trans. Automat. Control 33 (1988) 659-668.

[16] Y. Yamamoto, S. Hara, Relationships between internal and external stability for infinite-dimensional systems with applications to a servo problem, IEEE Trans. Automat. Control 33 (1988) 1044-1052.
[17] M.C. Smith, On stabilization and the existence of coprime factorizations, IEEE Trans. Automat. Control 34 (1989) 1005-1007.

[18] D. Youla, J. Bongiorno, C. Lu, Single-loop feedback stabilization of linear multivariable dynamical plants, Automatica 10 (1974) 153-173.

[19] A. Quadrat, On a general structure of the stabilizing controllers based on stable range, SIAM J. Control Optim. 42 (2004) 2264-2285.

[20] S. Gümüşsoy, Coprime-inner/outer factorization of SISO time-delay systems and FIR structure of their optimal $\mathrm{H}$-infinity controllers, Int. J. Robust Nonlinear Control 22 (2012) 981-998.

[21] C. Foiaş, H. Özbay, A. Tannenbaum, Robust Control of Infinite Dimensiona Systems: Frequency Domain Methods, Springer-Verlag, London, 1996.

[22] A. Tannenbaum, Modified Nevanlinna-Pick interpolation and feedback stabilization of linear plants with uncertainty in the gain factor, Internat. J. Control 36 (1982) 331-336.

[23] J.A. Ball, J.W. Helton, Interpolation with outer functions and gain equalization in amplifiers, in: Proc. MTNS 1979, 1979.

[24] C. Ganesh, Synthesis of optimal control systems with stable feedback, in: Ph.D. Thesis, Rice University, 1987. http://scholarship.rice.edu/handle/1911/16056.

[25] G. Gu, P.P. Khargonekar, E.B. Lee, Approximation of infinite-dimensional systems, IEEE Trans. Automat. Control 34 (1989) 610-618.

[26] J.-H. Moon, M.-N. Lee, M.J. Chung, Repetitive control for the track-following servo system of an optical disk drive, IEEE Trans. Control Syst. Tech. 6 (1998) 663-670.

[27] C. Cosner, G. Anwar, M. Tomizuka, Plug in repetitive control for industrial robotic manipulators, in: Proc. IEEE ICRA 1990, 1990

[28] Y. Yamamoto, S. Hara, Internal and external stability and robust stability condition for a class of infinite dimensional systems, Automatica 28 (1992) 81-93.

[29] Y. Yamamoto, Equivalence of internal and external stability for a class of distributed systems, Math. Control Signals Systems 4 (1991) 391-409.

[30] S. Gümüşsoy, H. Özbay, On the mixed sensitivity minimization for systems with infinitely many unstable modes, Systems Control Lett. 53 (2004) 211-216.

[31] K. Kashima, Y. Yamamoto, On standard $H^{\infty}$ control problems for systems with infinitely many unstable poles, Systems Control Lett. 57 (2008) 309-314.

[32] J.R. Partington, C. Bonnet, $H_{\infty}$ and BIBO stabilization of delay systems of neutral type, Systems Control Lett. 52 (2004) 283-288. 